12 Ateenyi-Agaba C. Conjunctival squamous cell carcinoma associated with HIV infection in Campala, Uganda [Letter]. Lancet 1995; 345: 695-6.

13 Byrne MA, Taylor-Robinson D, Munday $P$ Harris JRW. The common occurrence of human papillomavirus infection and intraepithelial neoplasia in women infected by HIV. AIDS 1989; 3: 379-82.

\section{Presumed ocular larva migrans presenting with features of diffuse unilateral subacute neuroretinitis}

EDITOR,-Ocular infection by Toxocara typically presents as diffuse endophthalmitis or a granuloma; less frequent are anterior uveitis, pars planitis, optic neuritis, conjunctivitis, keratitis, or lens involvement. ${ }^{1}$ Rarely, a motile larva may be visualised.

We present a patient with positive aqueous serology to Toxocara species associated with features consistent with diffuse unilateral subacute neuroretinitis (DUSN).

CASE REPORT

A 49-year-old man presented with a 3 day history of blurred vision of the right eye, associated with a floater and a right sided headache.

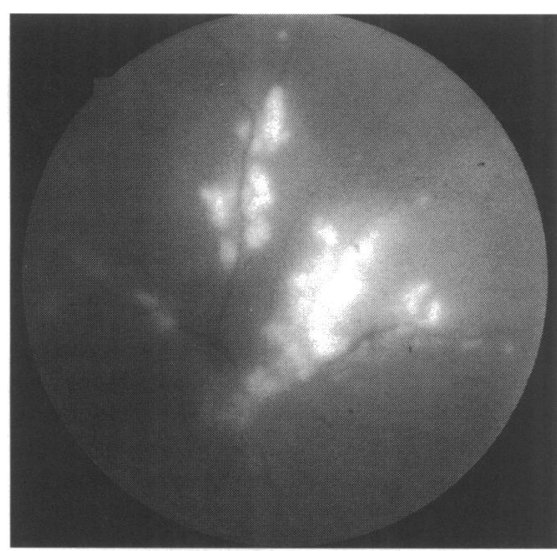

Fig $1 A$

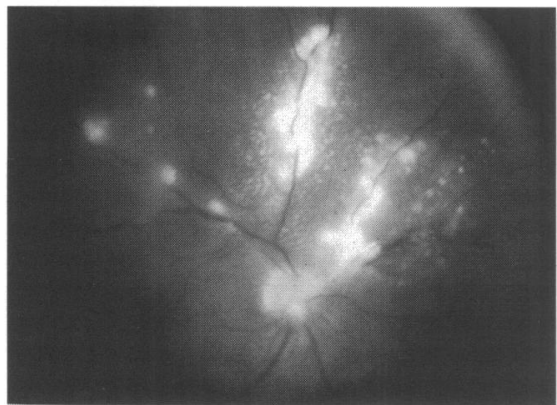

Fig $1 B$

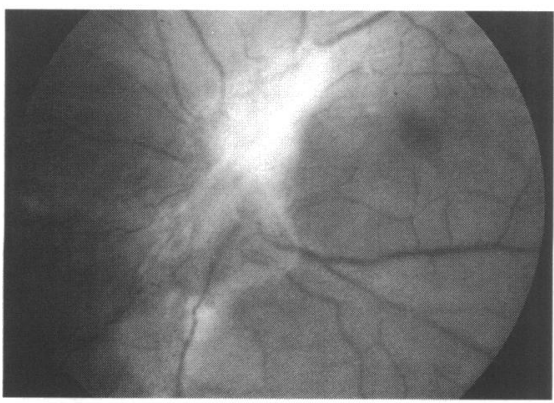

Fig $1 C$

Figure 1 (A) Initial presentation with 'string of pearls' lesion. (B) Crops of perivenous white plaques present during first week after presentation. (C) Slight preretinal fibrosis 1 month after presentation.
Two weeks previously right corneal trauma had resulted in a red eye which had failed to respond to topical chloramphenicol.

At presentation, the anterior chamber exhibited circumcorneal injection, aqueous cells, and flare with multiple posterior synechiae. A hazy fundus view revealed perivascular 'white patches' and macular oedema. Prednisolone acetate $1 \%$ hourly, atropine $1 \%$ three times daily, and flurbiprofen $100 \mathrm{mg}$ twice daily were prescribed initially.

A $2 \mathrm{~mm}$ hypopyon formed 24 hours later but disappeared the following day. Numerous keratic precipitates were present inferiorly. A white 'string of pearls' lesion appeared adjacent to the optic disc (Fig 1A). Intense photophobia impaired fluorescein angiography and initial fundus photography. There was an end systolic aortic murmur. Endogenous endophthalmitis was thus initially thought to be the probable diagnosis. Serological tests excluded systemic candidosis; antimicrobials were withheld pending laboratory findings.

Temporal progression of the disease is illustrated in Figure 2. Cytomegalovirus, herpes simplex virus, varicella zoster virus, Toxoplasma, Treponema pallidum, Mycobacterium tuberculosis, and sarcoid were excluded. Over the following 7 days visual acuity improved and the anterior chamber became less inflamed. The retinal changes
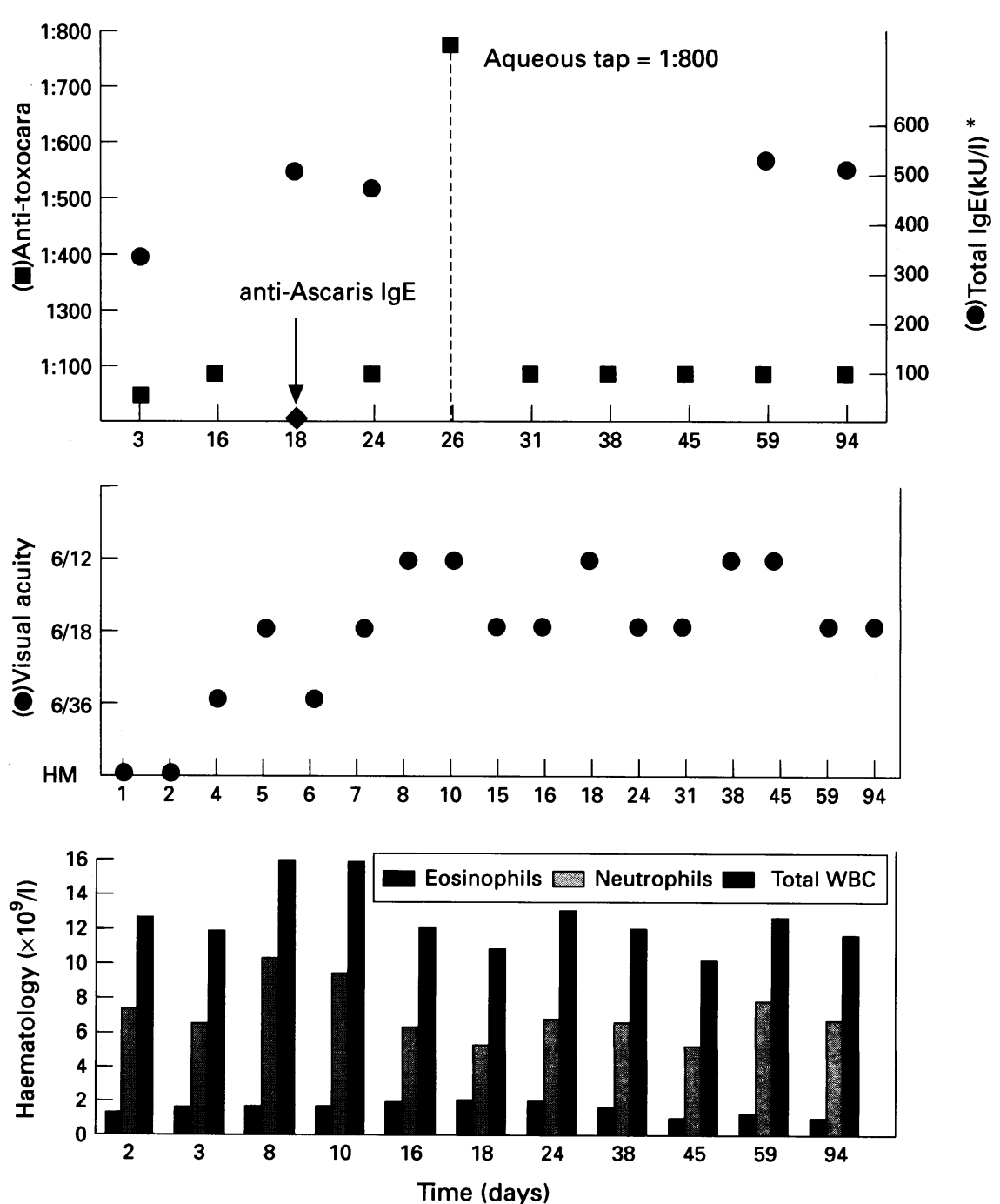

Figure 2 Clinical and laboratory observations. ${ }^{\star} I g E$ levels against house dust mite, grass pollen, cat and dog danders were negative or only slightly raised (Pharmacia CAP System). Specific IgE against Toxocara spp was not detected; parasitic helminth infection may cause polyclonal stimulation of IgE producing $B$ lymphocytes. Anti-Ascaris IgE was minimally raised at $0.35 \mathrm{U} / \mathrm{ml}$ (Pharmacia CAP System). Mebendezole $100 \mathrm{mg}$ was administered on day 15; E vermicularis was absent from second stool sample, day 59.

continued to evolve. Venous sheathing associated with a branch vein retinal occlusion plaques; this resembled DUSN ${ }^{2}$ (Fig 1B) Raised serum IgE and eosinophilia suggested yielded both Entamoeba coli cysts and Enterobius vermicularis ova.

the following 2 weeks the retinitis (1) auiescent, although elevated serum atypical clinical appearance ocular larva migrans was suspected. Enzyme linked immunosorbent assay (ELISA) ${ }^{3}$ on paired serum and aqueous samples demonstrated significantly elevated aqueous antibody idence of acute visceral larva migrans.

ment was discontinued after 1 month preretinal fibrosis developed around the optic disc (Fig 1C). Right visual acuity remained at $6 / 18$ for the ensuing 3 months.

COMMENT

DUSN is a clinical syndrome. Early feature of grey-white retinal lesions ${ }^{2}$ There is progressive visual loss, optic atrophy, and diffuse pigment epithelial degeneration. The aetiology of 
DUSN is undetermined, but has been attributed to subretinal migration by nematodes, including Toxocara. ${ }^{2}$ The clinical appearances described here are compatible with early DUSN.

Antibody activity against Toxocara spp was demonstrated in serum at 1:100 and 1:800 in aqueous. The ELISA measured all antibody classes against diagnostic antigens from shed larval cuticle. ${ }^{3}$ The clinically apparent retinitis may have been due to initiation of an immune response to previously shed antigens. Despite detailed biomicroscopy no intact larvae were observed, although this cannot be discounted.

Uveitis has been described in patients with Entamoeba coli infection ${ }^{4}$; this is likely to be an epiphenomenon.

Another hypothesis is that previous Toxocara infection could have sensitised the retina to subsequent inflammatory episodes. Many cases of posterior uveitis are thought to be 'endogenous' being linked to MHC II autoantigen expression and autoimmunity. ${ }^{5}$ Such autoimmune posterior uveitis may produce vitritis, retinochoroidal infiltrates, retinal vasculitis, and macular oedema. These were features present in this patient.

Trauma to ${ }^{6}$ and infection of, the anterior eye can induce non-specific posterior inflammation. Autoantibodies against corneal antigens produced by local trauma can cross react with uveal tissue; indeed uveitis is associated with elevated levels of anticorneal autoantibodies. ${ }^{7}$ Thus, antecedent trauma related anterior inflammation may have initiated an autoimmune reaction to retinal constituents if this was a previously sensitised patient.

Further, the eosinophilia and elevated serum IgE could have produced a type 1 hypersensitivity reaction by degranulation of choroidal mast cells. Local recruitment of immune cells with lymphokine release may induce, for example, vasodilatation and increased expression of 'adhesion' molecules and MHC II, producing breakdown of the blood-retinal barrier and enhanced immunoreactivity. This could also have contributed to initiation of an immune response against retinal autoantigens or Toxocara. Cells in the aqueous were a feature with this patient. Aqueous cytology to assess eosinophilia may have proved useful. Eosinophilia is a feature of a number of ocular diseases. ${ }^{8}$ Limited quantity of sample, however, precluded such investigation as it was considered more appropriate to investigate the anti-Toxocara antibody titre.

In summary, the patient described presented with panuveitis. The major feature was retinal vasculitis. Despite the atypical clinical appearance, an immune response to Toxocara antigen was demonstrated in the aqueous. Uveitis may have occurred in response to previously deposited Toxocara antigens or as a consequence of sensitisation to an autoimmune process.

We are grateful to $\mathrm{Mr} A \mathrm{R}$ Hinson and $\mathrm{Dr} \mathrm{C}$ McSharry for conducting the immunological tests, to Mr J McCormick for the clinical photography, and to Dr F Ghanchi.

F B KINNEAR GN DUTTON

Tennent Institute of Ophthalmology, Tentern Infirmary, Glasgow G11 $6 N T$

H V SMITH

Scottish Parasite Diagnostic Laboratory
Stobhill Hospital, Glasgow $G 213 U W$
1 Shields JA. Ocular toxocariasis. A review. Surv Ophthalmol 1984; 28: 361-81.

2 Gass JDM. Inflammatory diseases of the retina and choroid. In: Gass JDM, ed. Stereoscopic atlas of macular diseases diagnosis and treatment. 3rd ed. Volume 2. St Louis: Mosby, 1987: 455-549.

3 Smith HV. Antibody reactivity in human toxocariasis. In: Lewis JW, Maizels RM, eds Toxocara and toxocariasis. Clinical, epidemiological and molecular perspectives. London British Society for Parasitology and Institute of Biology, 1993: 91-111.

4 Knox DL, Bayless T. Gastrointestinal and ocular disease. In: Mausolf FA, ed. The eye and systemic disease. In: Mausolf FA, ed. The eye and syste
disease. St Louis: Mosby, 1975: 274-90.

5 Forrester JV. Duke-Elder lecture: new concepts on the role of autoimmunity in the pathogenesis of uveitis. Eye 1992; 6: 433-46.

6 Kruit PJ, Broersma L, Van Der Gaag R, Kijlstra A. Clinical and experimental studies concerning circulating antibodies to corneal epithelium antigens. Doc Ophthalmol 1986; 64: 43-51.

7 Rosenbaum JT, Tammaro J, Robertson JE Jr. Uveitis precipitated by non-penetrating ocular trauma. Am f Ophthalmol 1991; 112: 392-5.

8 Trocme SD, Aldave AJ. The eye and the eosinophil. Surv Ophthalmol 1994; 39: 241-52.

\section{Candida endophthalmitis}

EDITOR,-Candida chorioretinitis is the most common cause of endogenous endophthalmitis with a characteristic clinical ocular appearance. ${ }^{1}$ Well known risk factors include major surgery, ${ }^{2}$ especially on the gastrointestinal tract, indwelling intravenous catheters, ${ }^{2}$ intravenous hyperalimentation, ${ }^{3}$ intravenous drug abuse, ${ }^{45}$ and systemic antibiotic therapy. ${ }^{1}$ Typical lesions can be found in both eyes in two thirds of symptomatic patients and the lesions are often multiple. ${ }^{1}$ If recognised early and before vision threatening retinal complications have developed, treatment with intravitrea ${ }^{6}$ amphotericin B and imidazoles is highly effective in eradicating the organism and preserving vision. ${ }^{7}$

\section{CASE REPORT}

A 26-year-old white male presented with a $2 \frac{1}{2}$ week history of left blurred vision. Three weeks previously he had undergone cholecystectomy for chronic cholecystitis, and 2 days postoperatively had become pyrexial. Five years earlier he had required almost complete excision of his small bowel as a result of a strangulated volvulus, necessitating total parenteral nutrition via a Hickman cannula. The postoperative fever was found to be due to Candida albicans infection of his feeding cannula which was removed with resolution of his fever. No systemic antifungal therapy was given and drug sensitivities were not obtained. A new Hickman cannula was reinserted 8 days later.

Examination revealed an afebrile, healthy young man with no external signs of infection. The best corrected visual acuities were $6 / 5$ right and $6 / 60$ left. There was a mild anterior uveitis in the left eye, the right anterior segment was normal, and the intraocular pressures were normal. Examination of the left fundus showed the presence of fluffy white preretinal and intraretinal infiltrates at the posterior pole extending into the posterior vitreous (Fig 1). The right posterior pole was healthy but there was a single white lesion in the inferonasal retina extending to the vitreous base (Fig 2). The clinical appearances in both eyes were considered typical of Candida endophthalmitis and management by left pars plana vitrectomy, bilateral intravitreal amphotericin $B$ injections, and intravenous fluconazole was undertaken.

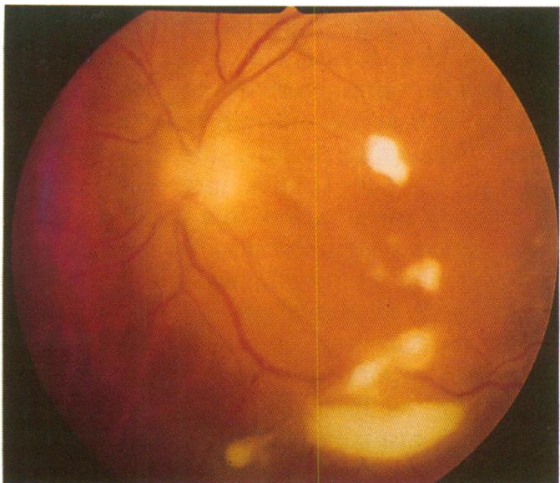

Figure 1 Fundus photograph of left eye posterior pole showing typical Candida lesions.

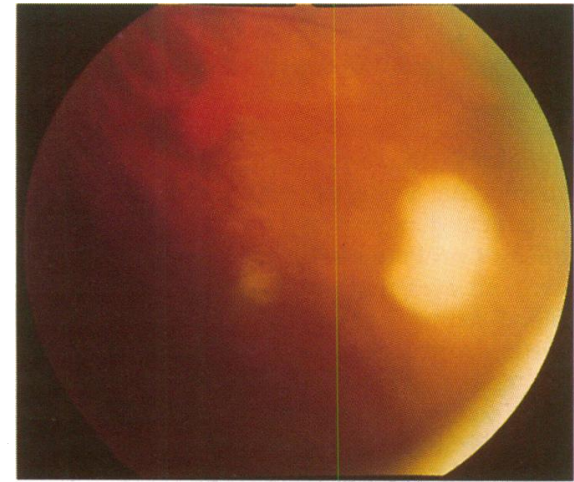

Figure 2 Fundus photograph of right eye showing peripheral lesion at presentation.

An uncomplicated left pars plana vitrectomy was performed with intracameral injection of $5 \mu \mathrm{g}$ amphotericin B in $100 \mu \mathrm{l}$ volume into both vitreous cavities. Intravenous fluconazole (200 mg daily) was commenced. Candida albicans was isolated from vitreous samples and was found to be sensitive to amphotericin B but resistant to fluconazole, so systemic therapy was changed to intravenous miconazole ( $600 \mathrm{mg}$ twice daily) and continued for the subsequent 6 weeks. Over this period, the left visual acuity steadily improved to $6 / 9$ at which level it has remained.

Despite an initial slight response in the right eye, the peripheral lesion enlarged and developed a localised shallow exudative retinal detachment. He also developed a severe maculopapular rash secondary to miconazole, which was stopped; further systemic therapy was considered unnecessary since he had already had 6 weeks of treatment. A right pars plana vitrectomy was then performed with injection of a further $5 \mu \mathrm{g}$ amphotericin B. No retinal breaks were identified at vitrectomy, and the subretinal fluid resorbed over the next month, leaving a pigmented scar. One year after his initial presentation, both eyes were quiet with no sign of disease activity, vision was $6 / 6$ right and $6 / 9$ left, and there was an inferonasal chorioretinal scar in the right eye.

COMMENT

This patient illustrates some of the considerable difficulties which can be encountered in managing Candida chorioretinitis. Although the clinical appearances of this disease are pathognomonic, delay in adequate treatment may still occur for a variety of reasons, which allows the opportunity for sight threatening complications such as retinal detachment or epiretinal membranes to 